\title{
A Survey on Junction Selection based Routing Protocols for VANETs
}

\author{
Dr. Irshad Ahmed Abbasi ${ }^{1 * *(M e m b e r ~ I E E E), ~ D r . ~ E l f a t i h ~ E l m u b a r a k ~ M u s t a f a ~}{ }^{2}$ \\ Department of Computer Science \\ Faculty of Science and Arts at Belgarn, University of Bisha \\ P.O. Box 60, Sabt Al-Alaya 61985, Kingdom of Saudi Arabia
}

\begin{abstract}
Objectives: To compare significant position-based routing protocols based on their underlying techniques such as junction selection mechanisms that provide vehicle-to-vehicle communications in city scenarios. Background: Vehicular Adhoc Network is the most significant offshoot of Mobile Adhoc Networks which is capable of organizing itself in an infrastructure-less environment. The network builds smart transportation which facilitates deriving in-terms of traffic safety by exchanging timely information in a proficient manner. Findings: The main features of vehicular adhoc networks pertaining to the city environment like high mobility, network segmentation, sporadic interconnections, and impediments are the key challenges for the development of an effective routing protocol. These features of the urban environment have a great impact on the performance of a routing protocol. This study presents a brief survey on the most substantial position-based routing schemes premeditated for urban inter-vehicular communication scenarios. These protocols are provided with their operational techniques for exchanging messages between vehicles. A comparative analysis is also provided, which is based on various important factors such as the mechanisms of intersection selection, forwarding strategies, vehicular traffic density, local maximum conquering methods, mobility of vehicular nodes, and secure message exchange. Application/Improvements: the outcomes observed from this paper motivate us to improve routing protocol in terms of security, accuracy, and reliability in vehicular adhoc networks. Furthermore, it can be employed as a foundation of references in determining literature that are worth mentioning to the routing in vehicular communications.
\end{abstract}

Keywords-Position-based; inter-vehicular; urban scenario; algorithms; reliability

\section{INTRODUCTION}

The Vehicular Adhoc Network (VANET) is a branch of Mobile Adhoc Networks (MANET). It is also called network on wheels which accomplishes communication between vehicles and among nearby vehicles. The vehicular nodes in VANETs are self-organized. They exchange information with each other in an infrastructure less environment [1-7]. VANET is a significant cost-effective tool for building an intelligent transportation system (ITS). It plays a vital role in traffic security and safety enhancement. It advances traffic management, and vehicles control. It is a significant way of providing the most recent applications to the on-wheel community. However, it is an outstanding challenge for the ITS industry to build vehicle to vehicle (V2V) and vehicle to infrastructure (V2I) interactions. The US FCC realises its intensifying benefits and allotted $75-\mathrm{MHz}$ spectrum for dedicated short-range communications (DSRC) for the deployment or exploitations of WLAN Technology for making vehicular communications a reality [1], [2], [5], [8], [10]. DSRC provides connectivity in a range of about one thousand meter [3], [8]. DSRC is an appropriate and vital technology for building vehicular communication. There are varieties of services that can be accomplished by using VANETs. These include accident avoidance, facilitating internet access inside vehicles, monitoring traffic flow regulations, locating parking lots, finding restaurants, and gas stations [29]. Inside vehicles, it is also useful in managing entertainment applications like playing games, watching movies, and listening music [10], [13].

The afore-mentioned applications cannot be accomplished without a competent routing protocol. The existing literature provides topology-based routing protocols and position-based routing protocols [3]. Topologies based routing protocols are ineffective in VANETs because of intermittent connectivity [13]. The position-based routing category is considered more effective in VANETs [10]. In position-based routing, particularly Junction selection-based routing is considered the most efficient routing mechanisms in city scenarios for addressing routing problems [3], [8], [9], [27], [29]. In the existing literature or surveys [7], [[11], [12], [15], [[17] only certain aspects like forwarding strategies or local optimum and mobility are considered. The study focuses on different methods of junction selection mechanism and their significance along with other aspects. The most prominent features or aspects of efficient routing protocols are tabulated. The working of different routing protocols is described with diagrams. It also provides some missing aspects like security, accuracy, and reliability which if added can further improve the latest junction selection-based routing protocols like DMJSR [28], and RPSPF [29]. Secure, accurate, and reliable exchange of messages is very important in VANETs for a message dissemination routing protocol.

The remaining portion of paper is arranged as follows. The vehicular adhoc network structural paradigm is elaborated in Section II. The detail about the position-based routing approaches particularly junctions selections based working in inter-vehicular communication environment for the urban scenarios is given in Section III. This section is also equipped with brief comparative analysis of position-based protocols from existing literature. The last Section IV concludes the paper and provides the future research directions.

* Corresponding Author 


\section{ARCHITECTURAL PARADIGMS OF VANETS}

In VANETs, each vehicle is equipped with devices called onboard units. Each onboard unit is enabled with wireless communication links and computational capabilities. Vehicles communicate through these wireless links. The vehicular nodes in VANETs act as members and as well as a router of the network. A node communicates directly with other nodes that are present inside its transmission range. The node uses an intermediate node for exchanging information with the nodes that are beyond its transmission range [4], [10], [27]. Due to self-organizing nature of VANET, its structural design categorized into three kinds: i) Pure adhoc networks ii) Pure cellular wireless local area network iii) Hybrid networks [12],[13].

Pure ad-hoc vehicular network design also named as intervehicle ad hoc network is presented in Fig. 1. It provides communication between vehicles and nearby vehicles. In this type of architecture, the collection and propagation of road associated information is carried out in the absence of any fixed infrastructure. Due to its infrastructure-less nature, it is cost-effective and easy to deploy [4], [14]. On the other hand, the vehicular nodes are set free to move at high speed. The highly mobile vehicular nodes frequently alter the network topological connections. The frequent topology changes cause network fragmentation [13]. In this kind of architecture, the network fragmentation due to high mobility makes routing of data more challenging [20], [28].

A cellular structural design of VANTs is provided in Fig. 2. It consists of cellular gateways and wireless access points which provide internet access to the vehicular nodes. The cellular architectural paradigm assists in giving information related to traffic jams and traffic flow control. Furthermore, it gives different types of other services which include data downloading, parking information, advertisement, and latest news [10], [24], [28]. It is very difficult to deploy because of the rising cost of cellular towers, geographical restrictions, and wireless access points [12], [14].

The hybrid structural design of VANETs is shown in Fig. 3. It is the mixture of both, infrastructure based domain and pure adhoc based domain. The adhoc domain furnishes $\mathrm{V} 2 \mathrm{~V}$ interactions. The infrastructure domain provides the V2I communications. This kind of architectural paradigm is supportive in giving more affluent content [8], [9]. It also provides better flexibility in contents sharing [26], [24].

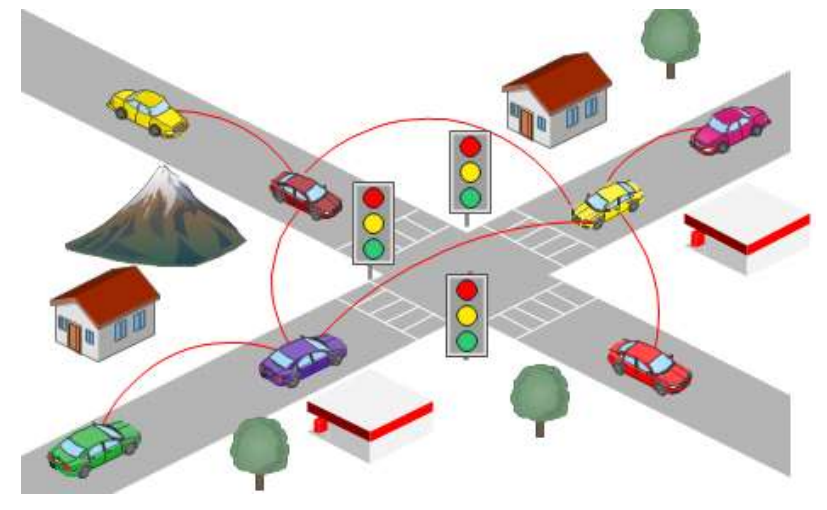

Fig. 1. Ad-hoc Networks Design.

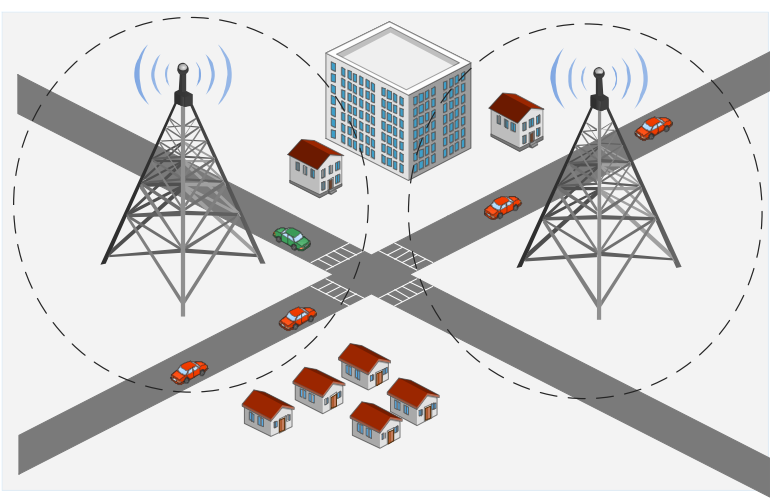

Fig. 2. Pure Cellular Design.

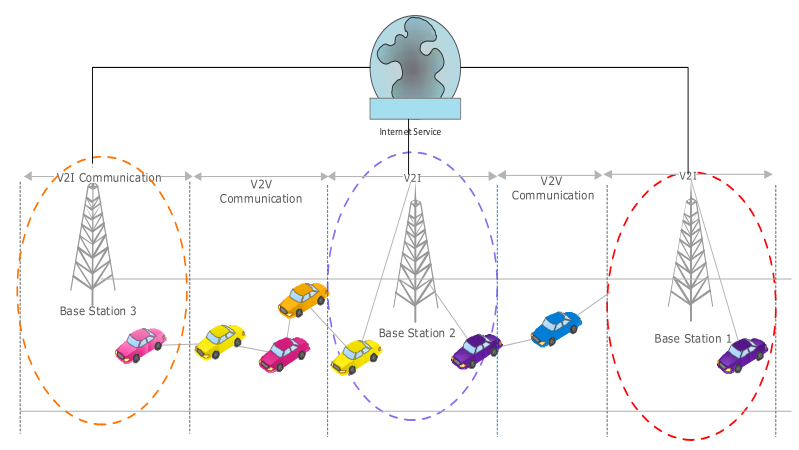

Fig. 3. Hybrid Design of VANETs.

\section{POSITION-BASED ROUTING IN VEHICULAR NETWORKS}

There are two general categories of baseline routing protocols in vehicular adhoc networks. Topology based routing protocols and position-based routing protocols [1]. The instances of topology based routing protocols include Adhoc On-distance Vector (AODV), Optimized Link State Routing (OLSR), and Dynamic Source Routing (DSR) [3]. In vehicular adhoc communication network, the protocols that belong to this category of routing are not feasible due to their path discovery mechanism [2], [19]. Also, their end to end routing path maintenance mechanism faces difficulties in VANETs. The reason is that irregular distribution of vehicular nodes and their highly mobile nature in adhoc domain cause regular path breakages in these routing protocols [9], [13], [21]. This increases routing overhead and makes VANETs ineffective. The second category of routing is position-based routing.

The existing literature shows that the position-based routing mechanisms are appropriate than topology based routing technique for handling routing problems in vehicular communications [8], [2-4], [13], [6], [17], [18]. The vehicular nodes in this category of routing protocols use their locations for communication. The communication between the source vehicular node and the destination vehicular node is either direct or through intermediate vehicular nodes. Each vehicular node in the network possesses GPS for locating its own position. When the source vehicle desires to interact with the destination vehicle, the source vehicle accomplishes its own position using GPS. The location of destination vehicle is established with the help of location services. The source vehicle or the intermediary vehicle keeps the latest positions of its one hop neighbors in its neighbor table using beacon 
exchanges. If the destination vehicle is inside the communication range of source vehicle, in this case, both directly communicate with one another. If the destination vehicle is outside of its direct reach than it relays the packet through an intermediary neighbor vehicle that is nearest to the destination node [8], [2-5], [21], [15]. In this way, the indirect communication between the source vehicular node and the destination vehicle is carried out by intermediate nodes.

Basically, the vehicular adhoc network has two environments. These are highway and urban environments [16]. The highway is composed of mainly straight and curvy roads having no obstacles. On the other hand, the urban environment contains streets with junctions. This environment is rich in impediments such as tall buildings.

The intersection of two or more streets is called junction. The packet passes through a set of junctions and relayed towards destinations. The obstacles around the streets and junction create problems in establishing an optimal routing path connecting source and destination [23]. As both the city and high environments have different structures and characteristics, the researchers designed protocols separately for each environment. In the existing literature, there are different types of position-based routing protocols. Fig. 4 presents the classification of position-based routing protocols. According to the figure, the position-based routing has two main classes i.e. urban environment based routing and highway environment based routing. The protocols are either proposed for $\mathrm{V} 2 \mathrm{~V}$ communications or V2I communications or for both the environments. This study mainly provides a brief description of routing schemes that are develop for $\mathrm{V} 2 \mathrm{~V}$ interactions in city scenarios. V2V based routings protocols are classified into two types i.e. static junction selection based routing protocols and dynamic junction selection based routing protocols. The dynamic junction selection based routing protocols are further classified into two classes i.e. dynamic one hop junction selection based routing protocols and dynamic multi hop junction selection based routing protocols [3], [17], [19], [28], [29]. A few of these routing proposals are traffic-aware while others are not. The protocols that are designed on the basis of traffic awareness concepts perform better in terms of packet delivery ratio, end to end delay, routing overhead and hop count as compared to other routing protocols [7], [9], [11], [12]. The description of V2V based routing protocols is given below.

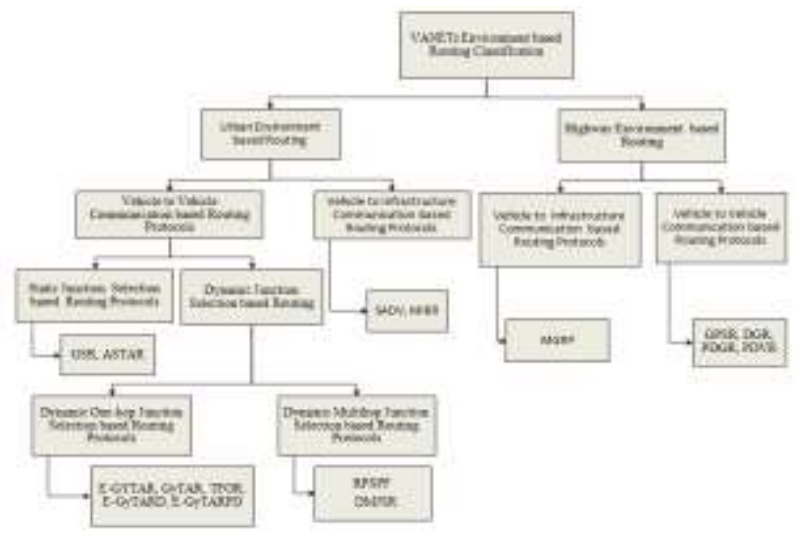

Fig. 4. Classification of Location based Routing Protocols.

\section{A. Greedy Perimeter Stateless Routing (GPSR) [22]}

GPSR is proposed for providing vehicle to vehicle interactions in a highway scenario. The description of GPSR provides us facts about the limitation of highway V2V based routing in a city scenario. GPSR finds source vehicle locations with the help of GPS. It locates neighboring nodes with the help of beacons exchange. The location service (likes GLS or HLS) [21] trace the location of the destination in this routing protocol. There are two working phases of GPSR. These are: i) the greedy phase, and ii) the perimeter phase. During greedy phase, the packet sender or forwarding node chooses its onehop neighbor that is the nearest to the destination for relaying packet towards the destination. The Greedy phase suffers from a local optimum problem that occurs if the forwarding node has no neighbor node that is nearest to the destination node than itself. The working of the greedy phase is presented in Fig. 5.

In this figure, Source vehicle $\mathrm{Sv}$ chooses neighbor vehicle $\mathrm{Bv}$ among its entire one-hop neighboring vehicles because of its nearest position to the target vehicle Dv and dispatches the packet to it. If GPSR meets the local optimum problem during the greedy mode, it overcomes this situation by using the perimeter phase. There are two steps in the perimeter phase. In the first step, the relative neighborhood graph (RNG) is used to accomplish graph planarization. In the second step, GPSR finds the next forwarding neighbor vehicular node by using right hand rule which is responsible for relaying packet toward destination. GPSR is ineffective in the city environment because of two main reasons. Initially, graph planarization fails due to impediments [3], [29]. Furthermore, the perimeter phase accomplishes long routing paths while dispatching packet towards a destination which causes an increase in the end to end delay. It also generates more routing overhead due to formation of routing loops [19], [18], [25].

Fig. 6 describes the operation of the perimeter phase. The source vehicle $S$ intends to communicate by sending a packet to destination vehicle $\mathrm{D}$. The source vehicle $\mathrm{S}$ forwards the packet to the neighbor vehicle A using greedy phase as it is closest to destination vehicle $\mathrm{D}$. At vehicle A, greedy phase stuck in local optimum as A itself is the nearest vehicular node to destination vehicle D as compared to all of its neighbors. The position of destination vehicle lies beyond the direct communication range of vehicle A. Vehicle A uses right hand rule of perimeter phase and overcomes this problem by choosing vehicle $X$ for forwarding packet. In the same way, at vehicle $X$ packet is sent to vehicle Y, GPSR will continue to use perimeter phase until it finds a vehicle to switch back to greedy phase.

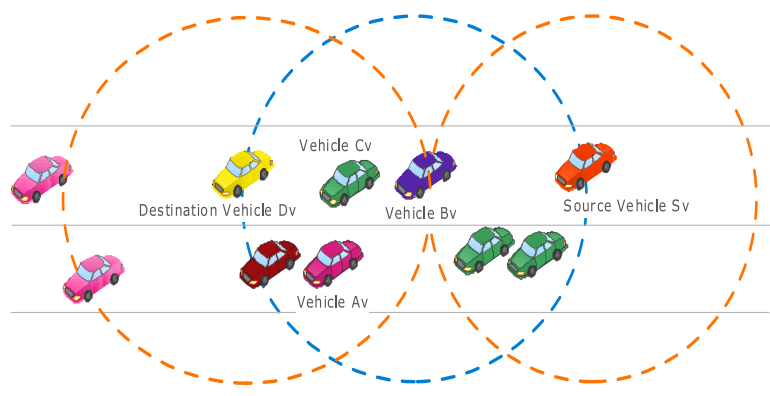

Fig. 5. Function of Greedy Mode. 


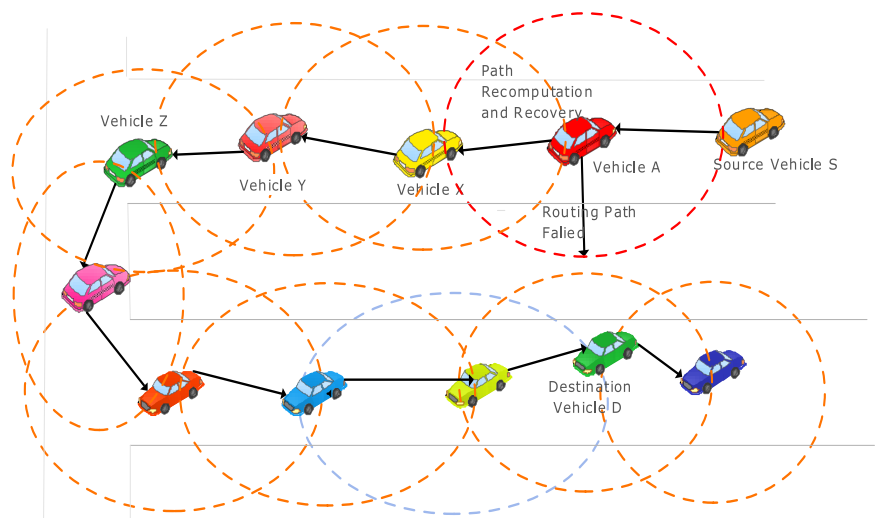

Fig. 6. Perimeter Mode of GPSR.

\section{B. Geographic Source Routing (GSR) Protocol [18]}

This protocol is developed for the urban setting to conquer the limitations of GPSR. It is position-based routing protocol. It accomplishes the position of destination vehicular node using reactive location service. It employs the Dijkstra shortest path algorithm to finds out the shortest route between source and destinations. The shortest route accomplished by GPSR consists of a sequentially arranged set of intersections. The packet moves from source vehicle to destination vehicle through the set of intersections. In between intersections, greedy forwarding is employed to forward the packet from one node to another node. The simulation results, with reasonable traffic density in city scenarios, shows that GSR outperformed the existing topology based DSR and AODV routing protocols pertaining to end-to-end delay and delivery ratio [9], [19]. This protocol is suffered from one main problem that it chooses intersections statically without the consideration of traffic density. It is not traffic aware. Traffic awareness in between junctions during junctions' selection is necessary, as it provides connectivity for moving packet towards destination $[3,8,28]$.

\section{Greedy Perimeter Coordinator Routing (GPCR) [25]}

It is developed for urban scenarios. This routing protocol is an integration of restricted greedy forwarding phase and perimeter phase. It does not use a digital city map. In the restricted greedy phase, the concept of coordinator node is introduced which is in charge of making routing decisions. The node located at the intersection is named as the coordinator node. Fig. 7 shows the working of the restricted greedy forwarding strategy.

According to this strategy, it is compulsory for the packet carrier node to select a coordinator at the junction for forwarding a packet. It bounds the packet carrier node to avoid packet forwarding to those nodes that are present across the junctions. Restricted greedy forwarding phase sometimes trapped in local optimum problem. GPCR overcomes this issue by applying the perimeter phase. In this phase, it is supposed that the city environment has natural planner graphs. Unlike GPSR, it ignores graph planarization. Making a planer graph in the city environment split the network into parts. The perimeter phase employs right-hand-rule to dispatch packet toward destination. There are certain demerits of this routing protocol. The restricted greedy phase always stops packet at the junction and increase the number of hop counts as compared to simple forwarding which deteriorate the performance of the network [14]. Fig. 8 shows the ineffectiveness of restricted greedy forwarding. The perimeter phase in GPCR delays in relaying packet towards a destination which diminishes the network performance [8, 2 and 11]. It is also not a traffic-aware routing protocol [3].

Fig. 7 demonstrates that vehicle A receives a packet from vehicle B. If vehicle $A$ uses greedy forwarding, it communicates the packet to vehicular node $\mathrm{C}$ that is nearest to the destination. If vehicle A uses restricted greedy forwarding, it sends the packet to a coordinator vehicle, located at the junction instead of vehicle $\mathrm{C}$.

The incompetence of restricted greedy forwarding is demonstrated in Fig. 8. In this figure, the source vehicle is marked with yellow color and the destination vehicle is marked with blue color. In case of simple greedy forwarding, the packet passes just 13 hops while traveling from its source vehicle to the required destination. On the other hand, in case of restricted greedy forwarding, it takes 17 hops for the packet to be transferred to the destination. This proves that restricted greedy forwarding is ineffective in terms of the number of hop counts [10].

\section{Anchor-based Street and Traffic-Aware Routing (A-STAR)}

\section{[23]}

This protocol is a position-based routing protocol. It accomplishes an anchor path by using statistically rated maps contain information pertaining to urban bus routes. The anchor path is based on connectivity. The packet passes through the anchors and relayed towards the destination. In the case of local optimum, it uses a route recovery strategy for the formation of a fresh path based on anchors. The outcomes of simulation and analysis indicate that A-STAR gives better performance than GSR and GPSR. The main reason is its competency of establishing an end-to-end route accomplishes connectivity even in low traffic density scenarios. However, its routing paths follow anchor path based on long city bus routes that may not be optimal and result in greater delays [3], [9], [13], [19].

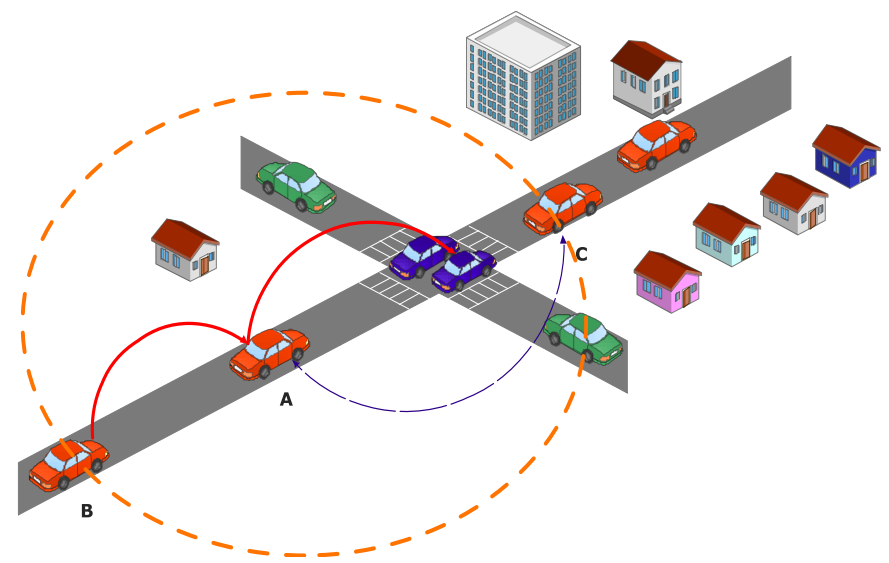

Fig. 7. Restricted Forwarding Mechanism of GPCR. 


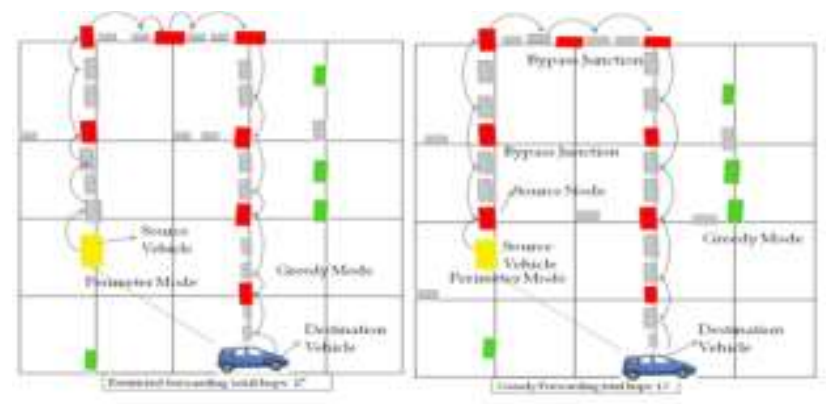

Fig. 8. Ineffectiveness of GPCR.

\section{E. Greedy Traffic-Aware Routing (GyTAR) Protocol [9]}

It is a position-based routing strategy that accomplishes optimal routes in city scenarios for relaying packet toward destination. It contains two phases. These are: i) dynamic junction selection method, and ii) an improved greedy forwarding technique for forwarding route in between junctions. In junction/intersection selection method, it decides the subsequent junction considering vehicular traffic density and the shortest distance to the destination. Its dynamic junction selection method moves the packet through city streets providing connectivity and the shortest distance to the destination. GyTAR outperforms the previous routing schemes like GSR and GPSR in terms of end to end delay, routing overhead, and packet delivery ratio. The main drawback of this routing protocol is that during the selection of the next junction, it ignores the vehicular nodes direction. Due to this, it suffers from the local optimum problem in some city scenarios which degrade the network performance [8].

\section{F. Enhanced Greedy Traffic-Aware Routing Protocol (E- GyTAR) [8]}

This protocol is an enhancement of GyTAR. It comprises of pair of modes. These are dynamic junction selection mode and an improved greedy forwarding for forwarding packets in between junctions. The dynamic junction selection mechanism selects the subsequent junction based on directional traffic density and the shortest routing path to the destination and thereby route the packet. Sometimes, its improved greedy forwarding stuck in local optimum situation. It exploits carryand-forward scheme to conquer this problem. The main negative aspect of E-GyTAR is that on multi-lane roads, it ignores non-directional traffic density. In case of absence of directional density, it is unable to select the next junction and the packet cannot be relayed towards the required destination node. The consideration of non-directional traffic density is very important in such scenarios for relaying packet towards the destination [3].

\section{G. Dynamic Multiple Junction Selection based Routing Protocol (DMJSR) [28]}

DMJSR is composed of multiple junction selection mechanism. The difference between DMJSR and existing approaches is its new dynamic multiple intersection selection method. Its novel junction selection mechanism establishes route by considering multiple junctions and thereby route data towards the required destination vehicle. It employs an enhanced greedy forwarding that maintains one-hop neighbor information instead of two-hops between the junctions.
DMJSR outperformed the existing one hop junction selection based routing schemes such as TFOR and E-GyTAR in case of packet delivery ratio and the end to end delay. The main dilemma associated with DMJSR is that its forwarding strategy ignores the link reliability while forwarding the packet. It is unable to sustain the frequent link ruptures caused by high speed vehicular nodes which degrade the network throughput [29].

In Fig. 9, the dynamic multiple junction selection technique of DMJSR routing protocol is presented. According scenario presented in the figure, the source vehicle $S$ is present at the current Junction $\mathrm{J}_{1}$. $\mathrm{J}_{1}$ has three two-hop neighbor junctions $\mathrm{J}_{4}$, $\mathrm{J}_{5}$ and $\mathrm{J}_{7}$. The source vehicle $\mathrm{S}$ selects two hop neighbor junctions $\mathrm{J}_{4}$ instead of $\mathrm{J}_{5}$ and $\mathrm{J}_{7}$ because of its higher traffic density which provides more connectivity and thereby dispatches the packet to the required destination vehicle D.

\section{H. Traffic Flow Oriented Routing Protocol (TFOR) [3]}

It is a position-based routing approach for the city surrounding. It accomplishes the routing path based on traffic flows. It has two modes: i) the junction selection mode which is based on the concentration of traffic density and shortest path. ii) A forwarding technique that maintains two-hop neighbor information. It selects the next junction on the basis of directional and as well as non-directional traffic flows. If directional traffic flow on the multi-lanes road is missing, it uses non-directional flow for routing the packet towards the destination. It concentrates on the urban streets containing higher traffic flows because higher traffic flows offer more connectivity in relaying packet towards a destination which enhances the network performance. Simulation results based on a realistic traffic city environment indicate that TFOR achieves higher performance in terms of packet delivery ratio and the end to end delay as compared to GSR, E-GyTAR, and GPSR. The problem with this routing protocol is that its improved greedy forwarding mode suffers from sudden link rupture problem. Also, its dynamic one-hop junction selection mechanism suffers from a local optimum problem at street level [28].

In Fig. 10, the intersection selection technique of trafficflow oriented routing protocol is presented. According to the figure, the source vehicular node is present at the current intersection $\mathrm{J}_{1} . \mathrm{J}_{1}$ has two neighbor intersections $\mathrm{J}_{2}$ and $\mathrm{J}_{3}$. The source vehicle node selects intersection $J_{3}$ instead of $J_{2}$ because of its higher traffic density and the shortest distance to the destination vehicle and thereby route the packet towards the destination.

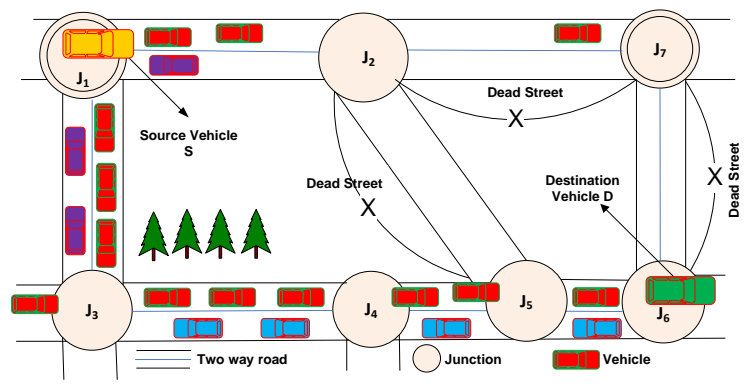

Fig. 9. DMJSR Junction Selection. 


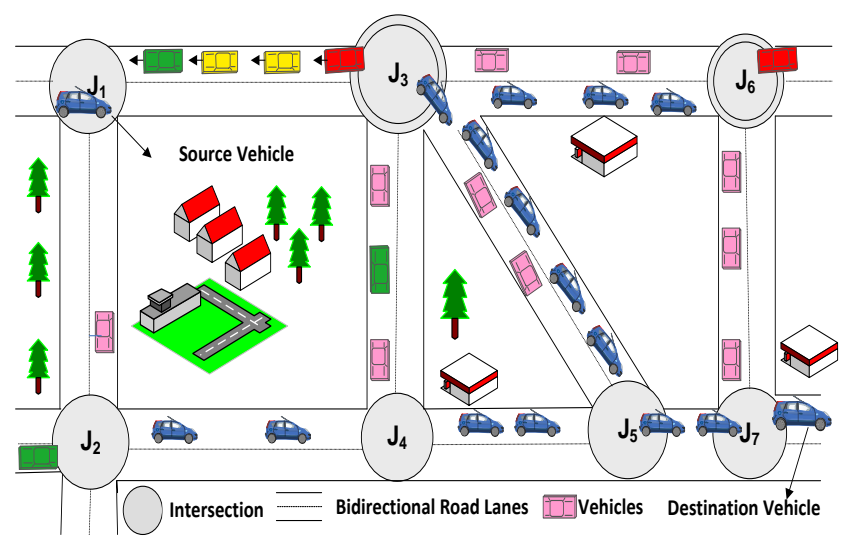

Fig. 10. TFOR Intersection Selection.

\section{Reliable Path Selection and Packet Forwarding Routing Protocol (RPSPF) [29]}

RPSPF is composed of multiple intersection selection mode and reliable packet forwarding mode. The difference between RPSPF and DMJSR is its novel reliable packet forwarding mechanism. It accomplishes route by considering multiple junctions and thereby route the packet towards destination. Its reliable packet forwarding approach forwards the packets to the next neighbor based on link life-time and link stability to avoid packet-drops because of rapid link ruptures. Simulation outcomes exhibit that RPSPS performs better than the existing one-hop junction selection based routing approaches like TFOR, GPSR, and E-GyTAR in terms of packet delivery ratio, end-to-end delay, and routing overhead. The problem with this routing protocol is that it cannot exchange message securely.

\section{J. Directional Geographic Source Routing (DGSR) [27]}

It is an improvement of GSR with a directional forwarding approach. This protocol consists of static junction/intersection selection mechanism of GSR. Instead of simple greedy forwarding, it uses a directional greedy forwarding strategy for forwarding packet in between the junctions. In this routing scheme, the shortest routing path between source and destination is accomplished based on the Dijkstra shortest path algorithm. The shortest routing path consists of a sequence of intersections. The packet passes through the sequence of intersections and reaches the destination. In the situation when this routing scheme suffers from local optimum problem, it uses a carry and forward approach. This protocol suffers from link sudden link rupture problems as its directional forwarding does not consider link reliability while forwarding [29].

\section{K. Enhanced Greedy Traffic-Aware Routing Protocol- Directional (EGyTAR-D) [27]}

In this routing scheme, E-GyTAR is enhanced with directional forwarding and named it as EGyTAR-D. It comprises of two phases. These are: i) the intersection selection phase and ii) the directional greedy forwarding phase. This protocol locates the position of destination vehicle using location service. Like E-GyTAR, It chooses the next junction considering directional traffic density and the shortest distance to the required destination. Directional forwarding is used to accomplish the forwarding of packets in between the junctions. Simulations results that consider city scenarios exhibit that EGyTAR improves packet delivery ratio and reduces the end to end delay as compared to DGSR and E-GyTAR. The directional forwarding of this protocol suffers from sudden link rupture problem [29]. Table I outlines the relative features of all the above mentioned inter-vehicular routing protocols.

TABLE I. COMPARATIVE FEATURES OF SignificANT Position-BASED Routing SCHEMES For City SCENARIOS

\begin{tabular}{|c|c|c|c|c|c|c|c|c|c|c|c|}
\hline \multirow{2}{*}{$\begin{array}{l}\text { Inter- } \\
\text { vehicular } \\
\text { Position } \\
\text { Aware } \\
\text { Unicast } \\
\text { Routing } \\
\text { Schemes } \\
\end{array}$} & \multicolumn{11}{|c|}{ Comparative Features } \\
\hline & $\begin{array}{l}\text { Secure } \\
\text { Message } \\
\text { Exchange }\end{array}$ & $\begin{array}{l}\text { Traffic } \\
\text { Density }\end{array}$ & $\begin{array}{l}\text { Static } \\
\text { Junction } \\
\text { Selection }\end{array}$ & $\begin{array}{l}\text { Dynamic } \\
\text { One hop } \\
\text { Junction } \\
\text { Selection }\end{array}$ & $\begin{array}{l}\text { Dynamic } \\
\text { Multi hop } \\
\text { Junction } \\
\text { Selection }\end{array}$ & $\begin{array}{l}\text { GPS } \\
\text { Require }\end{array}$ & $\begin{array}{l}\text { Digital } \\
\text { Map } \\
\text { Require }\end{array}$ & $\begin{array}{l}\text { Local } \\
\text { Optimum } \\
\text { Recovery } \\
\text { Technique }\end{array}$ & Reliability & $\begin{array}{l}\text { Hop } \\
\text { Count }\end{array}$ & $\begin{array}{l}\text { Realistic } \\
\text { Mobility } \\
\text { Flows }\end{array}$ \\
\hline GPSR[22] & 凶 & 凶 & - & 凶 & 凶 & $\nabla$ & $\nabla$ & $\begin{array}{l}\text { Perimeter } \\
\text { mode }\end{array}$ & 凶 & $\begin{array}{l}\text { One- } \\
\text { hop }\end{array}$ & $\nabla$ \\
\hline GSR[18] & 凶 & 凶 & $\nabla$ & 凶 & 凶 & $\nabla$ & $\nabla$ & $\begin{array}{l}\text { Switch back to } \\
\text { greedy } \\
\text { technique }\end{array}$ & 凶 & $\begin{array}{l}\text { One- } \\
\text { hop }\end{array}$ & $\nabla$ \\
\hline A-STAR[23] & 凶 & 凶 & $\nabla$ & 凶 & 凶 & $\nabla$ & $\nabla$ & $\begin{array}{l}\text { Anchor path } \\
\text { reconstruction }\end{array}$ & 凶 & $\begin{array}{l}\text { One- } \\
\text { hop }\end{array}$ & $\nabla$ \\
\hline GPCR[25] & 凶 & 凶 & - & 凶 & 凶 & $\nabla$ & 凶 & $\begin{array}{l}\text { Right hand } \\
\text { Rule }\end{array}$ & $凶$ & $\begin{array}{l}\text { One- } \\
\text { hop }\end{array}$ & $\nabla$ \\
\hline GyTAR[9] & 凶 & $\nabla$ & 凶 & $\nabla$ & 凶 & $\nabla$ & $\nabla$ & $\begin{array}{l}\text { Carry and } \\
\text { forward }\end{array}$ & 凶 & $\begin{array}{l}\text { One- } \\
\text { hop }\end{array}$ & $\nabla$ \\
\hline E-GTAR[8] & 凶 & $\nabla$ & 凶 & $\nabla$ & 冈 & $\nabla$ & $\nabla$ & $\begin{array}{l}\text { Carry and } \\
\text { Forward }\end{array}$ & 凶 & $\begin{array}{l}\text { One- } \\
\text { hop }\end{array}$ & $\nabla$ \\
\hline TFOR[3] & 凶 & $\nabla$ & 凶 & $\nabla$ & 凶 & $\nabla$ & $\nabla$ & $\begin{array}{l}\text { Carry and } \\
\text { Forward }\end{array}$ & $凶$ & $\begin{array}{l}\text { Two- } \\
\text { hop }\end{array}$ & $\nabla$ \\
\hline DGSR[27] & 凶 & $凶$ & $\nabla$ & 凶 & 凶 & $\nabla$ & $\nabla$ & $\begin{array}{l}\text { Carry and } \\
\text { Forward }\end{array}$ & 凶 & $\begin{array}{l}\text { One- } \\
\text { hop }\end{array}$ & $\nabla$ \\
\hline $\begin{array}{l}\text { D-EGyTAR } \\
{[27]}\end{array}$ & 凶 & $\nabla$ & 凶 & $\nabla$ & 凶 & $\nabla$ & $\nabla$ & $\begin{array}{l}\text { Carry and } \\
\text { Forward }\end{array}$ & 凶 & $\begin{array}{l}\text { One- } \\
\text { hop }\end{array}$ & $\nabla$ \\
\hline DMJSR[28] & 凶 & $\nabla$ & 凶 & 凶 & $\nabla$ & $\nabla$ & $\nabla$ & $\begin{array}{l}\text { Carry and } \\
\text { Forward }\end{array}$ & 凶 & $\begin{array}{l}\text { One } \\
\text { hop }\end{array}$ & $\nabla$ \\
\hline RPSPF[29] & $凶$ & $\nabla$ & 凶 & 凶 & $\nabla$ & $\nabla$ & $\nabla$ & $\begin{array}{l}\text { Carry and } \\
\text { Forward }\end{array}$ & $\nabla$ & $\begin{array}{l}\text { One } \\
\text { hop }\end{array}$ & $\nabla$ \\
\hline
\end{tabular}




\section{CONCLUSION AND FUtURE RESEARCH DiRECTIONS}

In this study, the most vital inter-vehicular communicationbased routing protocols designed for urban scenarios are presented. An overview of structural designs of the vehicular adhoc network is presented at the beginning of study. After that, a systematic discussion about the working of various position-based routing protocols along with their limitations is presented. It also presents a qualitative comparative investigation of the above-mentioned routing protocols based on the consideration of several significant parameters. These parameters include vehicular traffic density, forwarding strategies, mobility of vehicles, the mechanisms of junctions' selection which include static junction selection or dynamic one-hop junction selection or dynamic multi-hop junction selection, the techniques to handle local optimum situations, location services, and the ways of accomplishing the shortest routing path. There is a significant impact of all these parameters on the throughput of VANETs.

The designing of an effective optimal routing algorithm for an efficient inter-vehicular communication system faces several technical challenges. Even though, the routing of data for building an efficient ITS through VANETs received a lot of interest from worldwide wireless network research communities and organizations but yet there is a need for further vigilant investigation on some challenges associated with routing. For example, one of them is to design and develop a routing protocol that securely exchanges information in inter-vehicular communication system. The main and crucial component of VANETs is to have a protocol that is capable of quickly and timely disseminating accurate and secure messages about life intimidating incidents like traffic accidents and traffic jams. The dissemination of such critical messages in highly dynamic VANETs in the presence of malicious vehicular nodes is a challenging task. These malicious vehicular nodes normally temper the critical messages which result in devastating consequences in the form of collateral damage to neighboring vehicular nodes and drivers. With security and accuracy of messages, there is a need for reliability and stability of the links through which message travel to other vehicular nodes. In VANETs, high mobility of vehicular nodes makes the network intermittently connected. The intermittent connectivity induces sudden link breaks in the network at the time of forwarding or routing of packets. The induction of sudden link rupture increases packet loss which makes the network unreliable and ineffective. Inter-vehicular communication also needs a scheme of presenting accurate and well-timed information about the vehicular traffic density on the road. An optimal routing protocol that relays the packets considering the shortest distance and vehicular traffic density cannot be accomplished without an effective traffic density estimation mechanism. The traffic density determines the strength of connectivity in VANETs and the accomplishment of such a mechanism is also challenging. The vehicular communication system has two environments, highway, and city. Both have the different architectural designs. Developing a routing protocol that works in both the environment is another research challenge for the research communities.

In conclusion, the way of establishing a most robust routing path to the required destination determined by the mechanism exists in a protocol. However, for effective inter-vehicular communication, current routing protocols, unable to reflect properly the real-life city scenario characteristics. Therefore, VANETs need a routing protocol that is secure, reliable, stable, and accurately exchange the information between the vehicles. It must incorporate the actual-life urban environment characteristics like high mobility, intermittent connectivity, obstacles, dense and sparse nature of the networks to make VANETs effective in building an efficient transportation system.

\section{REFERENCES}

[1] A. Dahiya, R K. Chauhan, A Comparative study of MANET and VANET environment. Journal of Computing. 2010 July, 2 (7), pp. 8791.

[2] G. M. T. Abdalla, M. A. Abu-Rgheff, S. M. Senouci, Current Trends in Vehicular Ad Hoc Networks. Ubiquitous Computing and Communication Journal. UbiRoads. 2008 Mar, 6 (1), pp. 1-13.

[3] I. A. Abbasi., B. Nazir, S. A. Madani, A traffic flow-oriented routing protocol for VANETs. Springer EURASIP J. Wireless Communication and Networking. 2014 Jul, 121 (2014), pp. 1-17.

[4] F. Li, Y. Wang, Routing in vehicular ad hoc networks: A survey. Vehicular Technology, Magazine IEEE. 2007 Jun, 2 (2), pp. 12-22.

[5] J. Cheng, J. L. Cheng, M. C. Zhou, F. Liu, Routing in Internet of Vehicles: A Review. IEEE Transation on Intelligent Transportation System. 2016 Aug, 16 (5), pp. 1-20.

[6] B. T. Sharef, R A. Alsaqour, M. Ismail, Review: Vehicular communication adhoc routing protocols: A survey. Journal of Network and Computer Applications. 2014 Dec, 7 (3), pp. 63-96.

[7] S. A. Shah, J. Zhejiang, Unicast routing protocols for urban vehicular networks: review, taxonomy, and open research issues. Journal of Zhejiang University-SCIENCE (Computers \& Electronics). 2014, 15 (7), pp. 489-513.

[8] S. M. Bilal, S. A. Madani, I. A. Khan, Enhanced junction selection mechanism for routing protocol in VANETs. International Arab Journal of Information Technology. 2011 June, 8 (4), pp. 422-429.

[9] M. Jerbi, S. M. Senouci, R Meraihi, Y. G. Doudane, An improved vehicular ad hoc routing protocol for city environments. IEEE International Conference on Communication ICC07. 2007 Jun, pp. 3972 -3979 .

[10] S. M. Bilal, C. J, Bernardos, C. Guerrero, Position based routing in vehicular networks: A survey. Journal of Network and Computer Applications. 2013 Dec, 36 (2013), pp. 685-697.

[11] K. C. Lee, U. Lee, M. Gerla, Survey of Routing Protocols in Vehicular Ad Hoc Networks. In: Advances in Vehicular Ad-Hoc Networks, Developments and Challenges IGI Global. 2009, pp. 113-175.

[12] Y. W. Lin, Y. S Chen, S. L. Lee, Routing Protocols in Vehicular Ad Hoc Networks: A Survey and Future Perspectives. Journal of Information Science and Engineering. 2010 May, 26 (3) pp. 913-932.

[13] R. Sadakale, N. V. K. Ramesh, R. Patil, TAD-HOC Routing Protocol for Efficient VANET and Infrastructure oriented communication networks, Journal of Engineering, 2020 Jan, vol. 2020, pp. 1-12.

[14] J. Liu, J. Wan, Q. Wang, A survey on position based routing for vehicular ad hoc networks. Journal Telecommunications Systems archive. 2016 May, 62 (1), pp. 15-30.

[15] R. Sharma, A. Choudhry, Swami, An Extensive Survey on different Routing Protocols and Issue in VANETs. International Journal of Computer Applications. 2018 Nov, 106 (5), pp. 1-20.

[16] A. C. Silva, Applicability of position-based routing for VANET in highways and urban environment. Portugal Journal of Network and Computer Applications. 2013 Jul, 36 (2013), pp. 961-973.

[17] S. Dhankhar, S. A. Agrawal, Survey on Routing Protocols and Issues. International Journal of Innovative Research in Science, Engineering and Technology. 2014 June, 3 (6), pp. 1-15.

[18] C. Lochert, H. Hartenstein, J. Tian, H. Fubler, D. Hermann, M. A. Mauve, Routing strategy for vehicular ad hoc networks in city 
environments. Proceedings of IEEE Intelligent Vehicles Symposium. 2003, pp. $156-161$.

[19] A. Husain, R. Shringar, B. Kumar, and A. Doegar, Performance comparison topology based and position based routing protocols in Vehicular network environments. International Journal of Wireless \& Mobile Networks (IJWMN), 2019 Aug, 4 (3), pp. 1-16.

[20] M. M. Bhavani, A. Valarmathi, Smart city routing using GIS \& VANET system. Journal of Ambient Intelligence and Humanized Computing, 2020 Jun, 5(4), pp.1-9.

[21] W. Kieb, H. Fubler, J. Widmer, M. Mauve, Hierarchical location service for mobile ad-hoc networks. ACM SIGMOBILE Mobile Computing and Communications Review. 2004 Oct, 8 (1), pp. 47-58.

[22] B. Karp, H. T. Kung, and GPSR: Greedy perimeter stateless routing for wireless networks. Proceedings of the 6th annual international Conference on Mobile computing and networking USA. 2019, pp. 243254.

[23] C. Lochert, M. H. Mauve, H. Hartenstein, Geographic routing in city scenarios. SIGMOBILE Mobile Computing Commununication Review. 2005 Jan, 9 (1), pp. 69-72.

[24] U. Paranjothi, Y. Tanik, M .S. Khan, "Hybrid-Vehfog: A Robust Approach for Reliable Dissemination of Critical Messages in Connected
Vehicles, "Transactions on Emerging Telecommunications Technologies, vol. 30, pp. 1-11,2020

[25] B. C. Seet, G Liu, B. S. Lee, C.H. Foh, K. Wong, K. Lee, A-STAR: A Mobile Ad Hoc Routing Strategy for Metropol is Vehicular Communications. In Lecture Notes in Computer Science. NETWORKING 2004. International Conference on Research in Networking. 2004, pp. 989-999.

[26] N. Noorani, and S. A. H. Seno, SDN-and Fog Computing-based Switchable Routing Using Path Stability Estimation for Vehicular Ad Hoc Networks, Peer-to-Peer Networking and Applications, pp. 1-17, 2020.

[27] S. M. Bilal, A. R. Khan, \& S. Ali, Review and performance analysis of position based routing protocols. Springer Wireless Personal Area Communication. 2016 Aug, 1 (3), pp. 1-14.

[28] I. A. Abbasi, A. S. Khan, S. Ali, Dynamic Multiple Junction Selection Based Routing Protocol for VANETs in City Environment. Applied Sciences. 2018; 8(5):687.

[29] I. A. Abbasi, A. S. Khan, \& S. Ali, Reliable Path Selection and Packet forwarding Routing Protocol for VANETs Springer EURASIP J. Wireless Communication and Networking. 2018 Oct, 218 (2018), pp. 118. 